\title{
Metodología de cálculo de ETP aplicando teledetección caso Valle de Chincha
}

\author{
ETP calculation method using remote sensing application to the Chincha Valley
}

\author{
Cayo Leonidas Ramos Taipe ${ }^{1}$
}

\begin{abstract}
Resumen
El presente trabajo desarrolla el cálculo de la evapotranspiración (ETP) aplicando metodologías espacialmente distribuidas, como es el modelo SEBAL (Algoritmo para el Balance de Energía Superficial, "Surface Energy Balance Algorithm for Land"), basada en sensores remotos, que está compuesto por varios sub-modelos. Se desarrolló un caso de aplicación para el valle de Chincha en la costa del Perú en base a datos del satélite Landsat 5, registrado por el mapeador temático (TM), para el 12 de febrero del 2004. Los resultados obtenidos son representativos y sugieren que SEBAL puede ser considerado como un método viable para estimar ETP a nivel de valles o cuencas y además en zonas que posean limitada información.
\end{abstract}

Palabras clave: evapotranspiración; SEBAL; teledetección; imagen Landsat.

\begin{abstract}
This paper develops evapotranspiration calculation using spatially distributed methodologies, such as the SEBAL model (Algorithm for Surface Energy Balance, "Surface Energy Balance Algorithm for Land"), based on remote sensing, which is composed by several sub-models. We developed an application case for Chincha valley on the coast of Peru, with Landsat 5 satellite data, recorded by the thematic mapper (TM) for February 12, 2004. The results are representative and suggest that SEBAL can be considered as a viable method for estimating ETP on valleys or basins and also in areas having limited information.
\end{abstract}

Keywords: evapotranspiration; SEBAL; teledetection; Landsat image.

\section{Introducción}

La evapotranspiración (ETP) puede ser determinada experimentalmente, usando lisímetros, el balance hídrico en el suelo o puede ser estimada a partir de datos meteorológicos con aplicación de ecuaciones, como el Penman-Monteith (FAO-56). Sin embargo, dichas determinaciones son válidas para áreas homogéneas y cuando se requiere conocer la ET a escala regional o sobre grandes áreas. El uso de esas técnicas es prácticamente inaplicable o muy poco exacto. Por tanto, la aplicación de técnicas de sensor remoto basada en imágenes de satélites, se constituye en una alternativa de grandes potencialidades para enfrentar este tipo de requerimientos.

Varios algoritmos han sido desarrollados en los últimos años, dentro de estos destaca el algoritmo SEBAL ("Surface Energy Balance Algorithm for Land"), que fue desarrollado por Bastiaanssen en 1995, que permite la obtención de la evapotranspiración a través de datos de imágenes de satélite y algunos datos complementarios de superficie.

El objetivo del presente trabajo es aplicar un modelo de cálculo de la evapotranspiración distribuido espacialmente y el uso de sensores remotos en el valle de Chincha.

\section{Modelo SEBAL}

Es un modelo que permite calcular la evapotranspiración de áreas grandes con cualquier tipo de cobertura (vegetación, agua o descubiertas) a partir de imágenes de satélite. La evapotranspiración es calculada como la diferencia de la ecuación de balance de energía en base a píxeles.

$$
L E_{\text {PIXEL }}=\lambda E T_{\text {PIXEL }}=R_{\text {n.PIXEL }}-H_{n . P I X E L^{-}}-G_{n P I X E L}
$$

Donde $\mathrm{LE}_{\text {pixel: }}$ : flujo de calor latente del píxel, $\mathrm{ET}_{\text {pixel }}$ : evapotranspiración en cada píxel, 1: calor latente de

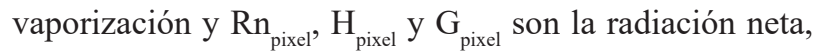
flujo de calor sensible y flujo de calor del suelo para cada píxel, respectivamente.

\section{Radiación neta $(\boldsymbol{R n})$}

La radiación neta es calculada como un balance de radiación entre las radiaciones netas de onda corta y onda larga en superficie. Se puede apreciar en la Fig. 1 que la radiación neta es el saldo de las entradas menos las salidas. Usualmente $\mathrm{R}_{\mathrm{n}}$ es positivo durante el día $\mathrm{y}$ negativo durante la noche. Una parte de esta energía neta es usada para la evaporación del agua del suelo, otra parte 
es usada para calentar el aire y el resto es almacenado en el suelo o en el cuerpo de agua.

\section{Flujo de calor del suelo $(G)$}

El flujo de calor del suelo $(G)$ representa el transporte vertical de calor, a través del proceso de conducción, de las capas superiores del suelo hacia las capas más profundas, en periodo diurno y de forma inversa en los periodos nocturnos. La estimación de G por el algoritmo SEBAL está determinada por la relación empírica propuesta por Bastiaanssen.

\section{Flujo de calor sensible $(\mathrm{H})$}

El flujo de calor sensible, $\mathrm{H}$, es la transferencia hidrodinámica del calor del aire, principalmente por convección, entre la superficie del suelo y las capas cercanas de aire.

SEBAL estima usando la ecuación propuesta por Brutsaert y relaciona la diferencia de la temperatura aerodinámica y la temperatura del aire.

\section{Flujo de calor latente (LE)}

El flujo de calor latente (LE) corresponde a la porción de energía de $\mathrm{Rn}$ que fue empleada en el proceso de evaporación de superficies y la transpiración de las plantas.

\section{Materiales y métodos}

En este trabajo se emplearon imágenes obtenidas por el Mapeador Temático de Landsat 5 compuestas por siete bandas espectrales, cuyas características están presentes en la Tabla 1, correspondiente a las T15:11:58Z del 12 de febrero de 2004. La Fig. 2 representa el área de estudio en composición RGB, combinación de las bandas 2, 4 y 5 .

\section{Localización del área de estudio}

El valle de Chincha, área del presente estudio, está situado en la parte baja de la cuenca del río San Juan, entre las latitudes $13^{\circ} 12^{\prime}-13^{\circ} 37^{\prime}$ Sur y longitudes $76^{\circ} 00^{\prime}-76^{\circ} 15^{\prime}$ Oeste. Políticamente, pertenece a la provincia de Chincha, departamento de Ica. Está constituido básicamente por un abanico fluvial de $25 \mathrm{~km}$ de ancho en su parte central, se extiende desde el nivel del mar hasta la cota $260 \mathrm{msnm}$, abarcando un área de $25,73 \mathrm{~km}^{2}$ y constituyéndose en una de las áreas agrícolas más importante de la costa peruana (Fig. 2).

La metodología aplicada es el algoritmo SEBAL, el cual calcula la evapotranspiración para cada píxel de la imagen por balance de energía. Rn es calculada con la ecuación 21 , a partir de mediciones satelitales de las bandas reflectantes y temperatura de superficie. G es estimado con la ecuación 22, con información de $\mathrm{Rn}$, temperatura de superficie y los índices de vegetación y $\mathrm{H}$ es estimado con la ecuación 23, con información de rangos de temperatura superficial, rugosidad superficial y velocidad del viento. Se presentan todas las etapas de cálculo de los diferentes componentes de balance de energía, para la obtención de LE y la evapotranspiración de 24 horas.

\section{Etapa 1: Calibración Radiométrica}

La calibración radiométrica o radiación espectral de cada banda $\left(\mathrm{L}_{\lambda}\right)$ representa la energía solar reflejada por cada píxel, se calcula según la ecuación:

$L_{\lambda}=\left(\frac{L_{M A X}-L_{M I N}}{255}\right) N D+L_{M I N}$

Donde ND es el número digital de cada píxel de la imagen (número entero de 0 a 255), $\mathrm{L}_{\mathrm{MIN}}$ y $\mathrm{L}_{\mathrm{MAX}}$ son las constantes de calibración espectrales mínimas y máximas que corresponden a las bandas de la imagen (ver Tabla 1).

\section{Etapa 2: Reflectancia monocromática}

La reflectancia monocromática de cada banda $\left(\rho_{\lambda}\right)$ está definida como un cociente entre el flujo de radiación reflejada y la radiación incidente. Se estima con la ecuación:

$o_{\lambda}=\frac{\pi L_{\lambda}}{E S U N_{\lambda} \cos \theta d_{r}}$

donde $\mathrm{L}_{\mathrm{li}}$ es la radiación espectral de cada banda, ESUN入 es la irradiación solar espectral de cada banda en el tope de la atmósfera ( $\mathrm{Wm}^{-2} \mu \mathrm{m}^{-1}$, Tabla 2), $\theta$ es el ángulo zenital solar $\mathrm{y}_{\mathrm{r}}$, es el cuadrado de distancia relativa Tierra-Sol (en unidades astronómicas - UA):

\section{Radiación de onda corta}

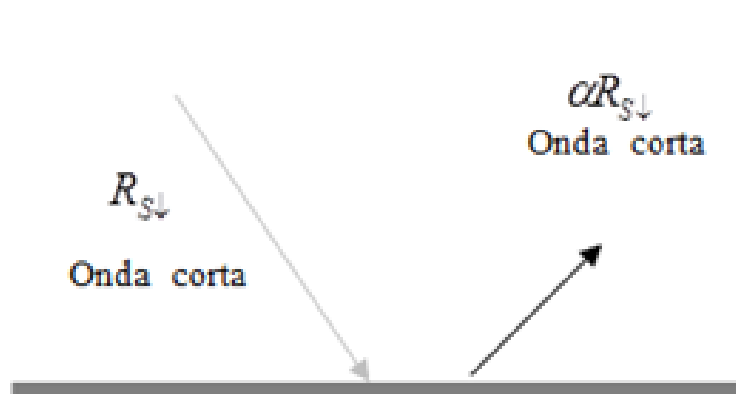

Radiación de onda larga

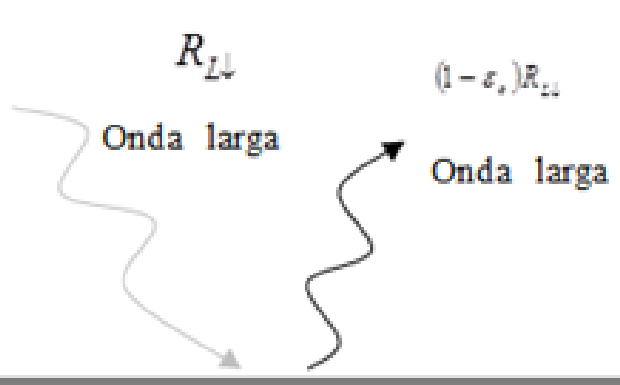

$R_{t}$

Onda larga

Superficie Vegetada 
$d_{r}=\left(\frac{r}{r_{o}}\right)^{2}=1,00011+0,034221 \cos \Gamma+0,00128 \operatorname{sen} \Gamma+0,000719 \cos 2 \Gamma+0,00077 \operatorname{sen} 2 \Gamma$

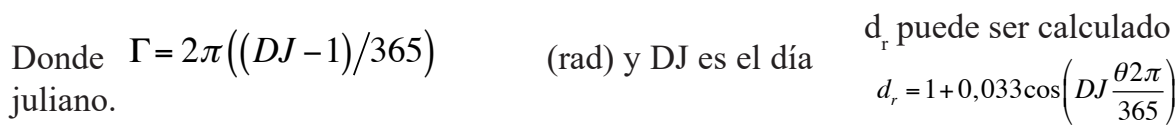

Tabla 1. Bandas del mapeador temático (TM) del Landsat 5, coeficientes de calibración e irradiaciones espectrales en el tope de la atmósfera

\begin{tabular}{|c|c|c|c|c|}
\hline \multirow{2}{*}{ Bandas } & \multirow{2}{*}{$\begin{array}{l}\text { Longitud de } \\
\text { onda } \\
(\mu \mathrm{m})\end{array}$} & \multicolumn{2}{|c|}{$\begin{array}{l}\text { Coeficiente de calibración } \\
\left(\mathrm{Wm}^{-2} \mathrm{sr}^{-1} \mu \mathrm{m}^{-1}\right)\end{array}$} & \multirow{2}{*}{$\begin{array}{l}\text { Irradiación espectral en } \\
\text { el tope de la atmósfera } \\
\qquad\left(\mathrm{Wm}^{-2} \mu \mathrm{m}^{-1}\right)\end{array}$} \\
\hline & & $\begin{array}{c}\text { a } \\
\text { Radiación mínima }\end{array}$ & $\begin{array}{c}\mathrm{b} \\
\text { Radiación máxima }\end{array}$ & \\
\hline 1 (azul) & $0,45-0,52$ & 191,6 & $-6,2$ & 1957 \\
\hline 2 (verde) & $0,53-0,61$ & 196,5 & -64 & 1828 \\
\hline 3 (rojo) & $0,62-0,69$ & 152,9 & $-5,0$ & 1557 \\
\hline 4 (IV-próximo ) & $0,78-0,79$ & 241,1 & $-5,1$ & 1047 \\
\hline 5(IV-medio ) & $1,57-1,78$ & 31,06 & $-1,0$ & 219,3 \\
\hline 6.1 (IV- termal) & $10,4-12,5$ & 17,04 & 0,0 & - \\
\hline 6.2 (IV-termal) & $10,4-12,5$ & 12,65 & 3,2 & - \\
\hline 7 (IV- medio) & $2,10-2,35$ & 10,8 & $-0,35$ & 74,52 \\
\hline 8 & & 243,1 & $-4,7$ & \\
\hline
\end{tabular}

Fuente: Imagen Landsat 12/02/2004.

Tabla 2. ESUN $\lambda$ de Landsat 5 TM (Markham y Barker, 1986), y Landsat 7 ETM + (Landsat 7 Ciencias de datos de usuario Manual Chap.11, 2002), ambos están en $\mathrm{W} / \mathrm{m}^{2} / \mu \mathrm{m}$

\begin{tabular}{cccccccc}
\hline & Banda 1 & Banda 2 & Banda 3 & Banda 4 & Banda 5 & Banda 6 & Banda 7 \\
\hline Landsat 5 & 1957 & 1826 & 1554 & 1036 & 215 & - & 80,67 \\
Landsat 7 & 1969 & 1840 & 1551 & 1044 & 225,7 & - & 82,07 \\
\hline
\end{tabular}

Fuente: Nelson, Allen, Tasumi, Trezza, Bastiaanssen, 2002.

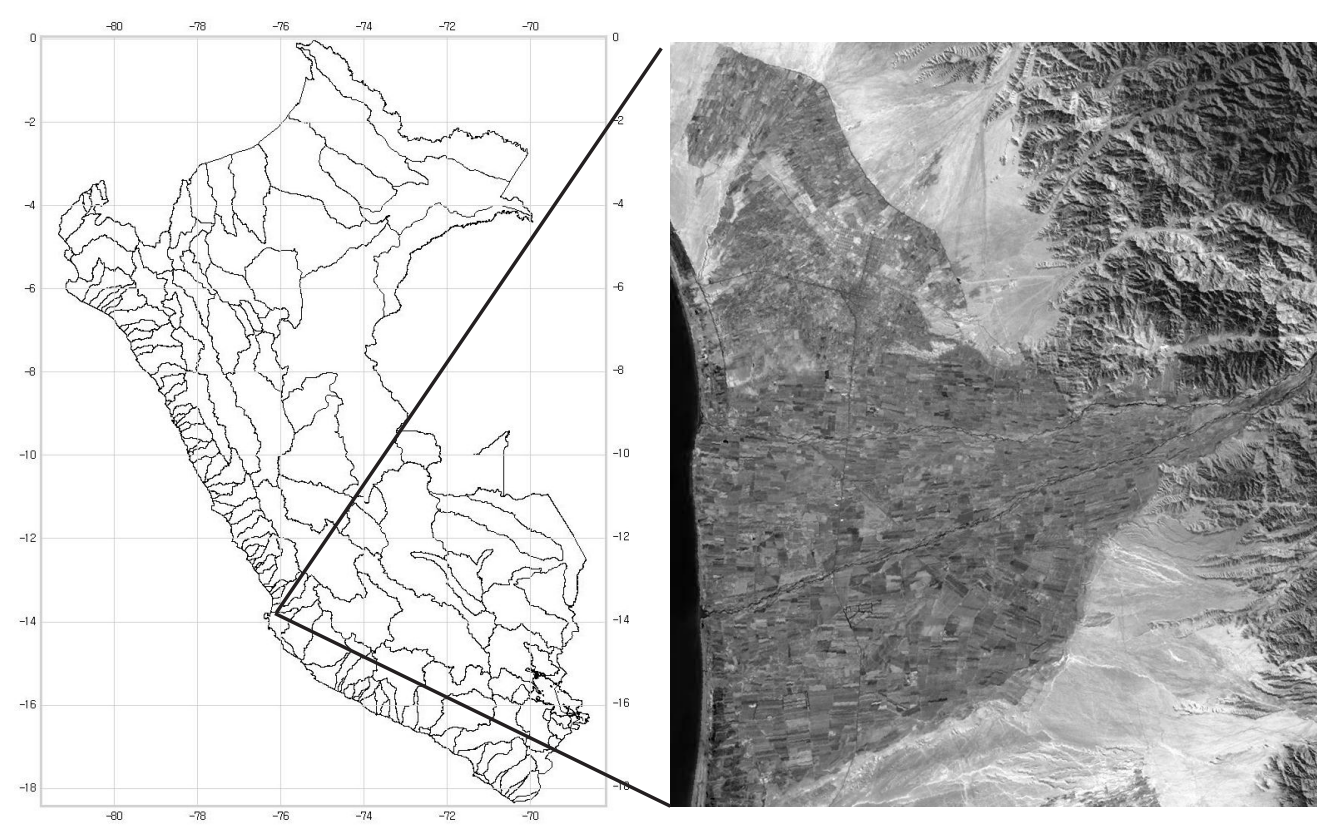

Figura 2. Valle de Chincha, parte baja de la cuenca del río San Juan (combinación de las bandas 2,4 y 5) 
Donde DJ es el día que se obtuvo la imagen (día juliano) y $\theta$ es el ángulo zenital solar

para el área estudiada $\mathrm{dr}=1,024 \mathrm{y} \operatorname{Cos} \Theta=0,853$

\section{Etapa 3: Albedo Planetario}

El cálculo del albedo planetario $\left(\alpha_{\text {toa }}\right)$, en el tope de la atmósfera, se calcula con la combinación lineal de las reflectancias espectrales $\left(\rho_{\lambda}\right)$.

$\alpha=\frac{\alpha_{t o a}-\alpha_{p}}{\tau_{s w}^{2}}$

\section{Etapa 4: Albedo de superficie}

El albedo de superficie es corregido por los efectos atmosféricos, usando la ecuación:

$\alpha_{\text {toa }}=\sum_{i=1}^{7}\left[\rho_{1, i} * w_{i}\right]$

donde $\alpha_{\text {toa }}$ es el albedo planetario, $\alpha_{\mathrm{p}}$ es la radiación solar reflejada por la atmósfera, que varía entre 0,025 y 0,04 , según SEBAL toma generalmente un valor promedio de $0,03 \mathrm{y} \mathrm{t}_{\mathrm{sw}}$ es la transmisividad atmosférica que para condiciones de cielo claro, puede ser obtenido por:

$\tau_{s w}=0,75+2 * 10^{-5} z$

Donde $\mathrm{z}$ altitud de cada píxel (m). Para efecto de simplificación, se utilizó $\mathrm{z}=70 \mathrm{~m}$ que representa la altitud media de la zona.

\section{Etapa 5: Índices de Vegetación: NDVI, SAVI y IAF.}

El Índice de Vegetación de Diferencia Normalizada (NDVI) es obtenido a través de la razón entre la diferencia de las reflectancias del infrarrojo próximo $\left(\rho_{\mathrm{IV}}\right)$ y el rojo $\left(\rho_{\mathrm{V}}\right)$ por la suma de las mismas:

$$
N D V I=\frac{\rho_{4}-\rho_{3}}{\rho_{4}+\rho_{3}}
$$

El NDVI es un indicador sensible a la cantidad y la condición de la vegetación verde. Sus valores varían de -1 a +1 y para superficies con alguna vegetación el NDVI varia de 0 a 1 , para el agua y nubes el NDVI generalmente es menor que cero. El Índice de Vegetación Ajustado para los efectos del Suelo (Soil Adjusted Vegetation índex SAVI), es un índice que busca armonizar los efectos de la naturaleza del suelo y la siguiente expresión permite estimarla:

$S A V I=(1+L) \frac{\rho_{4}-\rho_{3}}{L+\rho_{4}+\rho_{3}}$

donde L es constante. Para el presente estudio se utilizó L $=0,5$, que es el valor más frecuente en la literatura.
El Índice de Área Foliar (IAF) está definido por la razón entre el área foliar de toda la vegetación por unidad de área utilizada por la vegetación. El IAF es un indicador de la biomasa de cada píxel de la imagen. Se calcula con la siguiente ecuación empírica:

$I A F=-\frac{\ln \left(\frac{0,69-S A V I}{0,59}\right)}{0,91}$

\section{Etapa 6: Emisiones}

En la obtención de la temperatura de superficie, se emplea la ecuación de Planck invertida, válida para un cuerpo negro, donde la emisión es igual a 1. Como cada píxel no emite radiación electromagnética como un cuerpo negro, es necesario introducir la emisión de dominio espectral de la banda termal $\mathrm{e}_{\mathrm{NB}}$ para fines de computo de Ts, también el cálculo de la radiación de onda larga emitida por la superficie, requiere para su cálculo la emisión del dominio de la banda larga $\mathrm{e}_{0}(6-14 \mu \mathrm{m})$. Las emisiones $\mathrm{e}_{\mathrm{NB}} \mathrm{y} \mathrm{e}_{0}$ pueden ser obtenidas, para NDVI $>0$ y $\operatorname{IAF}<3$, con:

$$
\begin{aligned}
& \varepsilon_{N B}=0,97+0,00331 I A F \\
& \varepsilon_{0}=0,95+0,01 I A F
\end{aligned}
$$

Para píxeles con $\mathrm{IAF}>3, \mathrm{e}_{\mathrm{NB}}=\mathrm{e}_{0}=0,98$. Para cuerpos de agua $(\mathrm{NDVI}<0)$, en el caso de lagos y ríos, $\mathrm{e}_{\mathrm{NB}}=0,99 \mathrm{y} \mathrm{e}_{0}$ $=0,985$ (Allen et al., 2002).

\section{Etapa 7: Temperatura de superficie.}

Para la obtención de la temperatura de superficie $\left(\mathrm{T}_{\mathrm{s}}\right)$, se usa la radiación espectral de la banda termal $\mathrm{L}_{6}$ y la emisión $\mathrm{e}_{\mathrm{NB}}$ obtenida de la etapa anterior:

$$
\begin{aligned}
& T_{s}=\frac{1261}{\ln \left(\frac{\varepsilon_{N B}(60.8)}{L_{\lambda, 6}}+1\right)} \\
& \text { donde } L_{\lambda, 6}=0,0056322 D N+0,1238
\end{aligned}
$$

\section{Etapa 8: Radiación de onda larga emitida}

El tercer término del balance de radiación, es la radiación de onda larga emitida por la superficie $R_{L \uparrow}\left(\mathrm{Wn}^{-2}\right)$ y se obtiene a través de la ecuación de Stefan-BoItzman:

$$
R_{L \uparrow}=\varepsilon_{0} \sigma T_{s}^{4}
$$

donde $\mathrm{e}_{0}$ es la emisión de cada píxel, $\sigma$ es una constante de Stefan-BoItzman $\left(\sigma=5,67 * 10^{-8} \mathrm{Wm}^{-2} \mathrm{~K}^{-4}\right)$ y $\mathrm{T}_{\mathrm{s}}$ es la temperatura de la superficie $\left({ }^{\circ} \mathrm{K}\right)$.

\section{Etapa 9: Radiación de onda corta incidente}

La radiación de onda corta incidente $\mathrm{R}_{\mathrm{s} \downarrow}\left(\mathrm{Wm}^{-2}\right)$, segundo

Tabla 3. Valores de coeficientes de ponderación $w_{i}$

\begin{tabular}{cccccccc}
\hline & Banda 1 & Banda 2 & Banda 3 & Banda 4 & Banda 5 & Banda 6 & Banda 7 \\
\hline Landsat 5 & 0,293 & 0,274 & 0,233 & 0,157 & 0,033 & - & 0,011 \\
Landsat 7 & 0,254 & 0,149 & 0,147 & 0,311 & 0,102 & - & 0,036 \\
\hline
\end{tabular}


término del balance de radiación, se calcula por la siguiente ecuación:

$R_{S \downarrow}=G_{s c} \cos \theta * d_{r} \tau_{s w}$

donde $\mathrm{G}_{\mathrm{sc}}$ es una constante solar $\left(1367 \mathrm{Wm}^{-2}\right), \Theta$ es el ángulo zenital solar, $\mathrm{d}_{\mathrm{r}}$ es el inverso del cuadrado de la distancia relativa Tierra-Sol $\mathrm{y}_{\mathrm{sw}}$ es la transmisividad atmosférica. $\mathrm{R}_{\mathrm{s} \downarrow}$ puede ser considerado constante en toda el área de estudio, por ser pequeña.

\section{Etapa 10: Radiación de onda larga incidente}

La radiación de onda larga, incidente emitido por la atmósfera en la dirección de la superficie $\mathrm{R}_{\mathrm{s} \downarrow}\left(\mathrm{Wn}^{-2}\right)$, también puede ser calculada por una de las ecuaciones de Stefan-BoItzman:

$R_{L \downarrow}=0,85\left(-\ln \tau_{s w}\right)^{0.09} \sigma T_{a}^{4}$

$L_{\downarrow}=1,08\left(-\ln \tau_{s w}\right)^{0.265} \sigma T_{a}^{4}$

donde $\sigma$ es la constante de Stefan-BoItzman y $\mathrm{T}_{\mathrm{a}}$ es la temperatura del aire $(\mathrm{K})$ igual a la temperatura de la celda más fría $\mathrm{T}_{\text {frío }}$

\section{Etapa 11: Radiación neta}

La radiación neta en la superficie, como se ha mencionado, $\mathrm{Rn}\left(\mathrm{Wm}^{-2}\right)$ es calculada utilizando la siguiente ecuación de balance de radiación en la superficie:

$R_{n}=(1-\alpha) R_{S \downarrow}+R_{L \downarrow}+R_{L \uparrow}-\left(1-\varepsilon_{o}\right) R_{L \downarrow}$

Donde a: albedo de superficie para radiación de onda corta, $\mathrm{R}_{\mathrm{S} \downarrow}$ : Radiación de onda corta incidente, $\mathrm{R}_{\mathrm{L} \downarrow}$ : radiación de onda larga emitida por la superficie (termal), $\mathrm{R}_{\mathrm{L} \uparrow}$ : radiación de onda larga emitida por la atmósfera, $\mathrm{e}_{\mathrm{o}}$ : emisión termal de la superficie, y $\left(1-\mathrm{e}_{\mathrm{o}}\right) \mathrm{R}_{\mathrm{L} \downarrow}$ : parte de $\mathrm{R}_{\mathrm{L}}$ que es reflejado por la superficie.

\section{Etapa 12: Flujo de calor del suelo}

El flujo de calor del suelo $\mathrm{G}\left(\mathrm{Wm}^{-2}\right)$ puede ser obtenido con la ecuación empírica desarrollada por Bastiaanssen, para el cálculo del flujo de calor del suelo para cualquier condición de cobertura vegetal y tipo de suelo:
$G=\left[\frac{T_{s}}{\alpha}\left(0,0038 \alpha+0.007 \alpha^{2}\right)\left(1-0,98 N D V I^{4}\right)\right] R_{n}$

Donde G: flujo de calor del suelo, a: albedo de superficie, $\mathrm{T}_{\mathrm{s}}$ : temperatura de superficie $\left({ }^{\circ} \mathrm{C}\right), \mathrm{y}$ NDVI: índice de vegetación normalizada; estos valores normalmente se encuentran en el rango de 0 a 1 , cuando NDVI $>0,7$ representa una condición de cobertura completa de cultivos.

Para efecto de corrección de los valores del flujo de calor del suelo para cuerpos de agua $(\mathrm{NDVI}<0)$, puede ser utilizada la siguiente expresión: $\mathrm{G}=0,3 \mathrm{Rn}$ y $\mathrm{G}=0,5 \mathrm{Rn}$, según Allen et al., 2002.

\section{Etapa 13: Flujo de calor sensible}

El flujo de calor sensible $\mathrm{H}\left(\mathrm{Wm}^{-2}\right)$ es calculado en base a la velocidad del viento y temperatura de la superficie usando una calibración interna de diferencia de temperatura entre dos niveles próximos a la superficie, según la ecuación de Brutsaert 1982 (Bastiaanssen 1998a).

$H=\rho C_{p} \frac{T_{a e r o}-T_{a}}{r_{a h}}$

donde $\mathrm{r}$ : densidad del aire en función de la presión atmosférica; Cp: capacidad de calor especifico del aire; $\mathrm{T}_{\text {aero }}$ : temperatura aerodinámica de superficie; $\mathrm{T}_{\mathrm{a}}$ : temperatura del aire de referencia $\mathrm{y}_{\mathrm{ah}}$ : resistencia aerodinámica para el transporte de calor sensible entre la superficie y la altura de referencia.

SEBAL considera la diferencia de temperatura entre dos niveles próximos a la superficie, dT. El flujo de calor sensible, entonces, se define como:

$H=\rho C_{p} \frac{d T}{r_{a h}}$

donde $\mathrm{r}_{\mathrm{ah}}$ : resistencia aerodinámica para el transporte de calor entre $z_{1} y_{2}, y d T=T_{1}-T_{2}$ : diferencia de temperatura entre las dos alturas $z_{1}$ y $z_{2}$ sobre la superficie.

Para determinar el valor de dT para cada píxel, el procedimiento del modelo SEBAL asume la existencia de una relación lineal entre dT y la temperatura de superficie $\mathrm{T}_{\mathrm{s}}$ :

$d T=a T S+b$

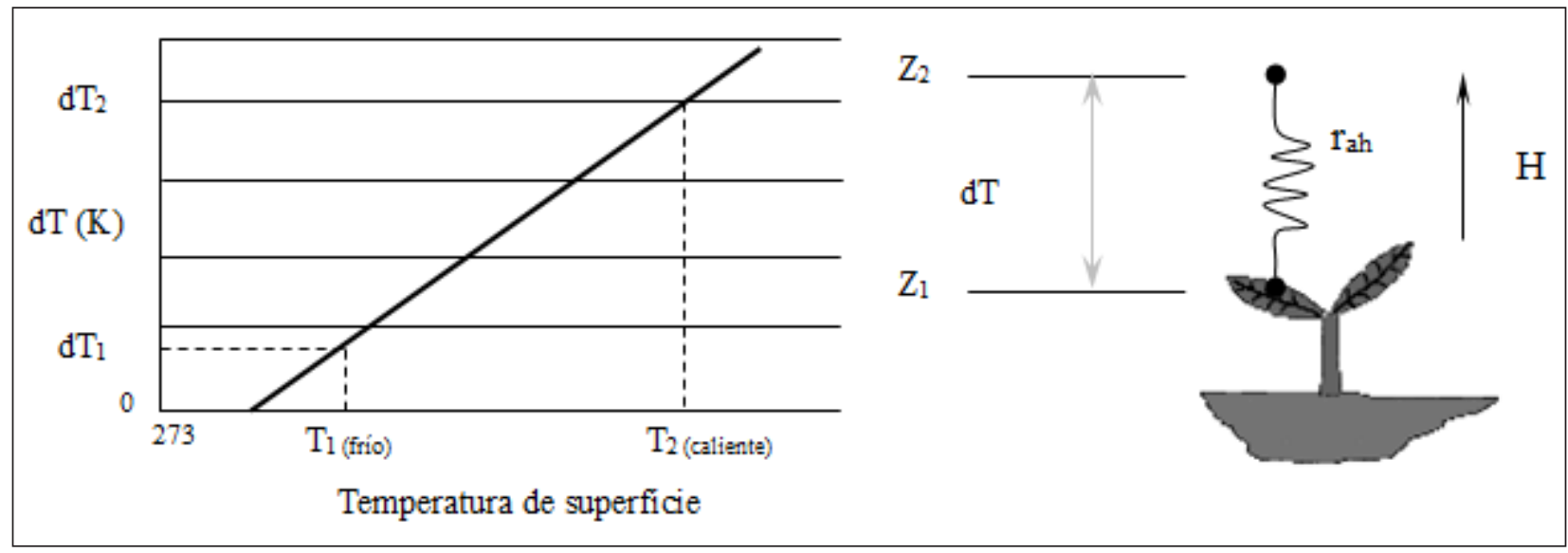


donde $\mathrm{T}_{\mathrm{s}}$ : temperatura radiométrica de superficie y "a" y "b": coeficientes empíricos que son obtenidos de los píxeles llamados "anclas" (Bastiaanssen, 1995). La implícita asunción en el modelo SEBAL es que "áreas calientes" (con emisión termal larga) crean una mayor dT vertical que en las "áreas frías" y que esta relación es lineal (Fig. 3).

El cálculo de $\mathrm{H}$ empieza con los datos de una estación meteorológica del interior de la imagen estudiada: la velocidad del viento $\left(\mathrm{ms}^{-1}\right)$ a nivel de $2 \mathrm{~m}$ y la altura media de la vegetación (m) circundante. Para la obtención de $\mathrm{H}$ con el modelo SEBAL, es necesario el conocimiento de dos píxeles, denominados "ancla", para determinar la variación de temperatura $(\mathrm{dT})$ y la resistencia aerodinámica de transporte de calor $\left(\mathrm{r}_{\mathrm{ah}}\right)$.

La primera etapa consiste en la obtención del coeficiente de rugosidad de superficie $Z_{0}$ de la estación que es calculado en función de la altura media de la vegetación (h), (Brutsaert, 1982).

$Z_{0}=0,12 h$

O las ecuaciones empíricas:

$Z_{0 \mathrm{~m}}=\exp (a N D I)+b$

donde $\mathrm{a}=0,9648$ y $\mathrm{b}=-3,3356$ son constantes, cuando se trabaja en bosques esta constante toma los valores de $\mathrm{a}=5,5895 \mathrm{y} \mathrm{b}=-3,2705\left(\mathrm{z}_{0}\right.$ puede tomar valores de $0,246 \mathrm{~m}$ o de hasta $4 \mathrm{~m}$ en bosques).

$\mathrm{Z}_{0 \mathrm{~m}}=\exp (\mathrm{a}+\mathrm{bSAVI})$

Donde: $\mathrm{a}=-5,809$ y $\mathrm{b}=5,62$

La resistencia aerodinámica $\mathrm{r}_{\mathrm{ah}}\left(\mathrm{sm}^{-1}\right)$ es calculada considerando que la atmósfera se encuentra en condición de estabilidad neutra por la siguiente expresión:

$r_{a h}=\frac{\ln \left(\frac{Z_{2}}{Z_{1}}\right)}{u_{*} k}$

donde $z_{1}$ e $z_{2}$ son las alturas en metros sobre la superficie (generalmente son utilizados $\mathrm{z}_{1}=0,1 \mathrm{y} \mathrm{z}_{2}=2,0 \mathrm{~m}$ ), $\mathrm{u}_{*}$ velocidad de fricción $\left(\mathrm{ms}^{-1}\right)$ y $\mathrm{k}$ es una constante de Von Karman (0,41).

Allen et al. (2002) recomiendan considerar $\mathrm{z}=0,30 \mathrm{~m}$, con el cual $\mathrm{z}_{0 \mathrm{~m}}=0,036 \mathrm{~m}$. La velocidad de fricción $\mathrm{u}_{*}\left(\mathrm{~ms}^{-1}\right)$ es calculada usando el perfil logaritmo del viento para la condición de estabilidad neutra:

$u_{*}=\frac{k u_{x}}{\ln \left(\frac{Z_{x}}{Z_{\text {om }}}\right)}$

donde $\mathrm{k}$ es la constante de Von Karman, u es la velocidad del viento $\left(\mathrm{ms}^{-1}\right)$ a una altura $\mathrm{z}_{\mathrm{x}}(2,0 \mathrm{~m}$, por ejemplo $)$ e $\mathrm{z}_{0 \mathrm{~m}}$ es el coeficiente de rugosidad (m).

Considerando la atmósfera en equilibrio neutro, la velocidad del viento $\mathrm{u}_{100}\left(\mathrm{~ms}^{-1}\right)$ a un nivel de $\mathrm{z}=100 \mathrm{~m}$, donde se asume que los efectos de la rugosidad de la superficie son despreciables, se estiman con la ecuación:

$u_{100}=u_{*} \frac{\ln \left(Z_{100} / Z_{0 m}\right)}{k}$
Considerando que $u_{100}$ es constante en toda la escena estudiada, puede ser calculada la velocidad de fricción $\mathrm{u}_{*}$ $\left(\mathrm{ms}^{-1}\right)$ para cada píxel, a través de la ecuación:

$u_{* 100}=\frac{k u_{100}}{\ln \left(z_{100} / z_{0 m}\right)}$

donde $Z_{0 m}(m)$ fue calculado en función de SAVI ecuación 28

El cálculo de la diferencia de temperatura próxima a la superficie dT $\left({ }^{\circ} \mathrm{C}\right)$ para cada píxel es calculada por SEBAL a través de una relación lineal entre $\mathrm{dT}$ y $\mathrm{T}$ (temperatura de superficie) expresado en la ecuación 25. El píxel "frío" se escoge en un área bien irrigada, donde se asume que el flujo de calor sensible es nulo $\left(\mathrm{H}_{\text {frio }}=0\right)$ y el flujo de calor latente $\lambda \mathrm{ET}_{\text {frio }}\left(\mathrm{Wm}^{-2}\right)$ está dado por:

$\lambda E T_{\text {frio }}=R_{n}-G$

A su vez el píxel "caliente" se selecciona en un área de suelo expuesto o desnudo, donde se asume que el flujo de calor latente es nulo $\left(\lambda_{\text {caliente }}=0\right)$ y el flujo de calor sensible $\mathrm{H}_{\text {caliente }}\left(\mathrm{Wm}^{-2}\right)$ está dado por:

$H_{\text {caliente }}=R n-G$

$d T=H_{\text {caliente }} r_{a h} / \rho_{a r} . c_{p}$

donde Ts, Rn, G y $\mathrm{r}_{\mathrm{ah}}$ son obtenidos del píxel caliente de la imagen. Teniendo estos valores se establece la relación lineal $\left(a+b T=r_{a h}(R n-G) / p C p\right)$. Como en el píxel frío $d T=$ 0 , o sea, $\mathrm{a}+\mathrm{bT},=0$, entonces se tiene un sistema de dos ecuaciones con dos incógnitas para el cálculo de a y b. Luego se obtiene H, según la ecuación 24.

Los valores encontrados no representan los valores más exactos de $\mathrm{H}$ para cada píxel y son usados como valores iniciales en un proceso iterativo, considerando efectivamente la condición de estabilidad de cada píxel, debido a los efectos turbulentos ocurridos en la superficie y que afectan las condiciones atmosféricas y la resistencia aerodinámica.

La longitud de Monin-Obukhov L (m) se emplea para definir las condiciones de estabilidad de la atmósfera y se calcula en función de los flujos de calor y de momentum con la siguiente expresión:

$L=-\frac{\rho C_{p} u_{*}^{3} T_{s}}{k g H}$

donde $\rho$ es la densidad del aire $\left(1,15 \mathrm{kgm}^{-3}\right), \mathrm{C}_{\mathrm{p}}$ es el calor especifico del aire, $\mathrm{u}$, es la velocidad de fricción en cada píxel de las imágenes $\left(\mathrm{ms}^{-1}\right), \mathrm{T}_{\mathrm{s}}$ es la temperatura de superficie $(\mathrm{K})$, g es la aceleración de la gravedad $\left(9,81 \mathrm{~ms}^{-2}\right)$ y $\mathrm{H}$ es el flujo de calor sensible $\left(\mathrm{Wm}^{-2}\right)$. Dependiendo de las condiciones atmosféricas, los valores de las correcciones de estabilidad para el transporte de momentum $\left(\mathrm{y}_{\mathrm{m}}\right)$ y de calor $\left(\mathrm{y}_{\mathrm{h}}\right)$ debe ser considerado. Para esto, se usan las fórmulas presentadas en Bastiaanssen (1995) y Allen et al. (2002):

El valor corregido para la velocidad de fricción $\mathrm{u}_{*}\left(\mathrm{~ms}^{-1}\right)$ está dado por:

$u_{*}=\frac{k u_{100}}{\ln \left(z_{100} / z_{0 m}\right)-\Psi_{m(100)}}$ 
donde $\mathrm{U}_{100}$ es la velocidad del viento a $100 \mathrm{~m}^{\left(\mathrm{ms}^{-1}\right), \mathrm{k} \text { es }}$ la constante de von Karman $(0,41), Z_{0 m}$, es el coeficiente de rugosidad de cada píxel (m) y $\mathrm{y}_{\mathrm{m}(100 \mathrm{~m})}$ es la corrección de estabilidad para el transporte de momentum a $100 \mathrm{~m}$.

Teniendo $\mathrm{u}_{*}$ corregido, se obtiene el valor corregido para la resistencia aerodinámica de transporte de calor $\mathrm{r}_{\mathrm{ah}}\left(\mathrm{sm}^{-1}\right)$ a través de la siguiente ecuación:

$r_{a h}=\frac{\ln \left(z_{2} / z_{1}\right)-\Psi_{h(z 2)}+\Psi_{h(z 1)}}{u_{*} k}$

donde $\mathrm{Z}_{2}=2,0 \mathrm{~m}, \mathrm{z}_{1}=0,1 \mathrm{~m}$, e $\mathrm{y}_{\mathrm{h}(\mathrm{z} 2)}$ é $\mathrm{y}_{\mathrm{h}(\mathrm{z} 1)}$ son las correcciones de estabilidad para el transporte de calor a $2,0 \mathrm{~m}$ y $0,1 \mathrm{~m}$, respectivamente.

\section{Etapa 14: flujo de calor latente}

El flujo de calor latente IET $\left(\mathrm{Wm}^{-2}\right)$ definido como el flujo de calor latente entregado por la superficie, debido a un proceso de evaporación o de evapotranspiración, se obtiene por diferencia entre la radiación neta, el flujo de calor del suelo y el flujo de calor sensible:

$\lambda E T=R_{n}-G-H \quad(38)$

donde lET es el valor del flujo de calor latente instantáneo.

\section{Etapa 15: Evapotranspiración actual 24 horas}

La evapotranspiración actual de 24 horas es calculada por la ecuación:

$E T_{24}=\frac{86400 \Lambda\left(R_{n 24}-G_{24}\right)}{\lambda}$

Donde, Rn24 es la radiación neta; G24 es el flujo de calor del suelo; 86,400 es el número de segundos en un periodo de 24 horas y 1 es el calor latente de vaporización $(\mathrm{J} / \mathrm{kg})$. El calor latente de vaporización permite obtener la ET24 en mm/day, L es la fracción evaporativa; G24 puede ser aproximado como cero en superficies con suelo y vegetación, esto debido a que la energía se almacena en el suelo durante el día y es regresada al aire en la noche. El valor para G24 de la neblina puede generalmente ser aproximado también como cero. Para un cuerpo de agua, especialmente en lagos profundos, G24 no se convierte en cero porque el agua tiene una alta capacidad de almacenamiento de calor.

Enel cálculo de G24 para el agua puede emplearse:

$\mathrm{G}_{24}=\mathrm{Rn}-100 \quad$ (40)

$\mathrm{G}_{24}=0,9 \mathrm{Rn}-50$

En la ecuación 40, el calor latente de vaporización 1 está definido como:

$\lambda=\left(2,501-0,00236\left(T_{o}-273\right)\right) * 10^{6}(\mathrm{~J} / \mathrm{kg})$

La siguiente ecuación permite calcular Rn24 bajo condiciones de cielo claro (todo el día):

$R_{n 24}=(1-\alpha) R_{a 24} \tau_{s w}-110 \tau_{s w}$ (43)

Donde $\mathrm{Ra} 24$ es la radiación diaria extraterrestre en la zona de estudio. La fracción de la evaporación L de cada píxel, útil para estimar la evapotranspiración de 24 horas, es considerado constante en todo el día y puede ser calculado con los valores instantáneos de la imagen con:

$\Lambda=\frac{L E}{R_{n}-G}$ (44)

La ecuación anterior puede ser descrita como:

$\Lambda=\frac{R_{n}-G-H}{R_{n}-G}$

Donde los valores para Rn, G y H son valores instantáneos obtenidos de la imagen. Las unidades para todos los parámetros de flujo están expresado como $\mathrm{W} \mathrm{m}^{2}$.

\section{Resultados y discusión}

El procesamiento digital de la imagen del satélite

Tabla 4. Parámetros instantáneos y diarios y flujos durante el paso de Landsat en los píxeles frío y caliente para 12 feb 2004

\begin{tabular}{llll}
\hline \multicolumn{1}{c}{ Ítem } & \multicolumn{1}{c}{ Frío } & \multicolumn{1}{c}{ Caliente } & \multicolumn{1}{c}{ Observación } \\
\hline Longitud & 8505335 & 8514353,16 & \\
Latitud & 373027,2 & 379279,9 & \\
T $(\mathrm{K})$ & 311.1546 & 315.2713 & Calculada de la banda 6 de landsat \\
Rn (W m-2) & 628.0643 & 583.7156 & Resultado del SEBAL \\
G (W m-2) & 127.5566 & 175.0579 & Resultado del SEBAL \\
Albedo & 0,1132 & 0,1379 & Calculado con bandas 1,2,3,4,5,7 landsat \\
IVDN & 0,7577 & 0,1348 & Calculado con bandas 3 y 4 landsat \\
IVAS & 0,6502 & 0,1145 & Calculado con bandas 3 y 4 landsat \\
IAF & 2,9621 & 0,0273 & Calculado con SEBAL \\
Superficie & Cub. algodón & suelo desnudo & Área agrícola \\
Kc & 0,8 & 0 & Tomado de FAO-56, algodón \\
ET mm/h & 0,53 & 0 & ET instantánea, ecuación de Penmman M. \\
LE $(\mathrm{W} \mathrm{m-2)}$ & 294.784 & 136.7344 & Calculado con SEBAL \\
H (W m-2) & 205.7242 & 271.9233 & Calculado con SEBAL \\
ET24 mm/d & 2,4913 & 0,1 & ET Calculado con SEBAL. \\
\hline
\end{tabular}


proporcionó como resultado las imágenes de temperatura superficial, albedo e índice de vegetación de la zona de estudio. Mediante la aplicación del algoritmo SEBAL se obtuvieron mapas de flujos de calor latente, sensible y de calor en el suelo y finalmente mapas de la fracción de evaporación y de evapotranspiración diaria.

En la Tabla 4 se muestra un resumen de la información relacionada con los píxeles frío y caliente, los valores instantáneos de ET fueron calculados y tomaron el valor $\mathrm{ET}=0,53 \mathrm{~mm} / \mathrm{h}$ para el píxel frío usando la ecuación de Pennman Monthei, para el momento en que el satélite tomó la imagen (15:11 GMT). La ET diaria para el píxel "frío" calculado con el modelo SEBAL toma el valor de $\mathrm{ET}=2,4913 \mathrm{~mm} / \mathrm{d}$. Los valores de radiación neta $\left(\mathrm{R}_{\mathrm{n}}\right)$, flujo de calor del suelo $(\mathrm{G})$ y flujo de calor sensible $(\mathrm{H})$ para ambos píxeles (frio y caliente); estos flujos son valores instantáneos correspondientes al momento en que pasó el satélite y los sensores TM tomaron la información.

La Fig. 4 muestra el mapa de evapotranspiración para todo el valle de Chincha, con resolución de $30 \times 30 \mathrm{~m}$. En ella se tiene una variación de los valores de ET en el rango de 0 , correspondiente a un suelo desnudo, a 4,9mm correspondiente a un suelo cubierto de vegetación al 100 $\%$ y con buena dotación de agua. El mapa de ET también incluye valores para las áreas cercanas con vegetación natural y vegetación riparian y no presentan ET en las zonas como la playa y/o zonas eriazas.

Todos estos valores de ET son importantes para el balance hidrológico del área así como para el modelamiento de las aguas subterráneas. La información de evapotranspiración para toda la imagen se muestra en la Tabla 5. La evaporación en el mar y los ríos se encuentra en el rango de 4,19 a 4,240mm, con un valor promedio de ET 4,22mm. Los valores de ET en la zona agrícola suministrados de agua básicamente por sistema de irrigación (mostrado en Fig. 4) toma valores en un rango de 0,6 (suelo sin cultivo) a $4,16 \mathrm{~mm}$ que representa la ET ocurrida en un campo cubierto plenamente por cultivos y recientemente regado. El valor promedio de ET para el área agrícola fue 2,43mm. Para un total de 26.809 ha de tierras agrícolas sembradas se tiene un volumen de $428,952 \times 10^{3} \mathrm{~m}^{3}$ de agua.

De acuerdo con la Tabla 5, el total de agua usado durante el día por evaporación en el mar y evapotranspiración en las áreas irrigadas fue de $550,434 \times 10^{3} \mathrm{~m}^{3}$. Este valor representa información básica para la gestión del agua en sistemas de riego, especialmente el valor de evapotranspiración de los cultivos y reservorios existentes.

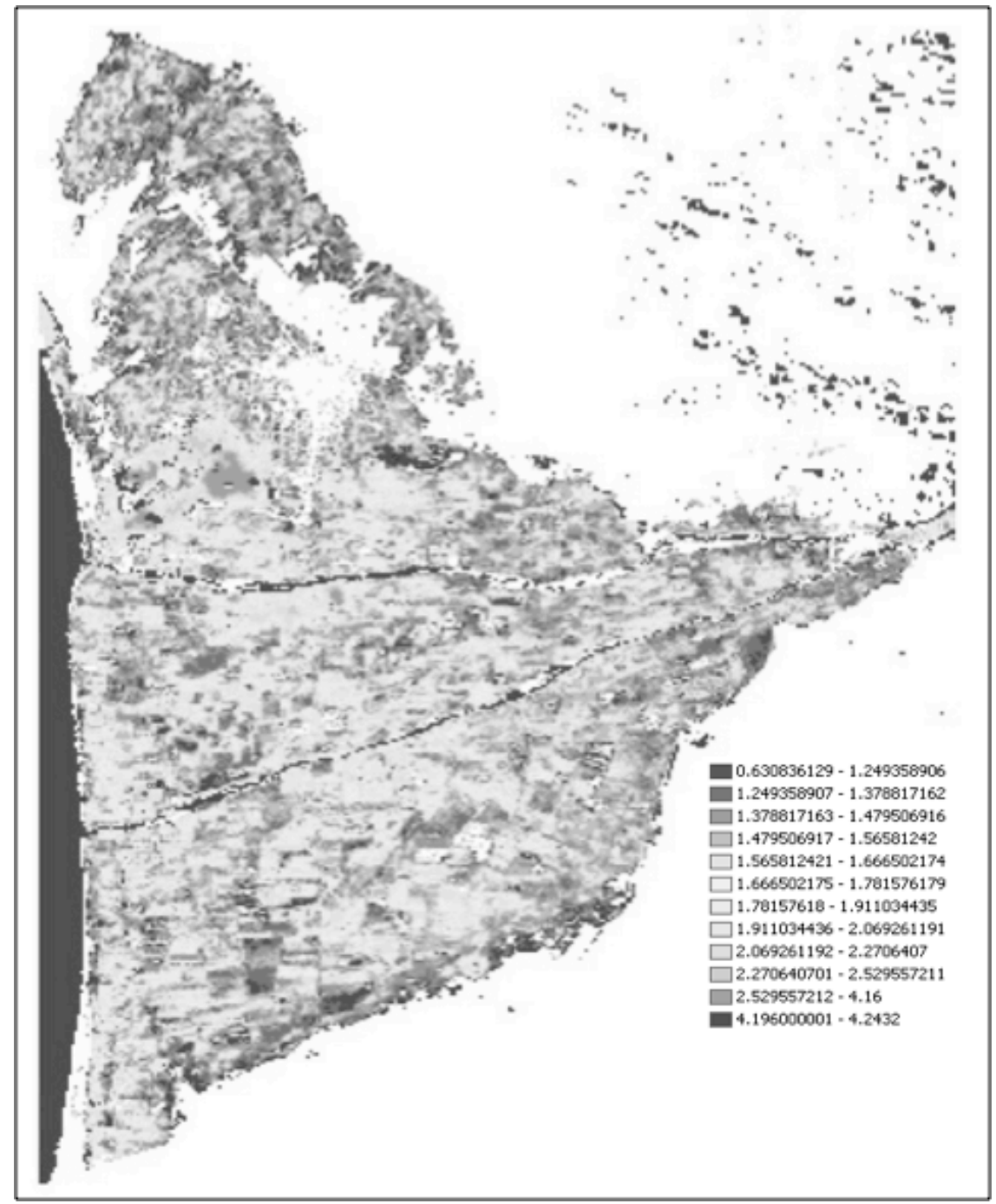

Figura 4. Mapa de evapotranspiración diaria (ET) para el 12 de febrero del 2004, para el valle de Chincha. El mapa tiene una resolución de $30 \mathrm{~m} \times 30 \mathrm{~m}$ 
Tabla 5. Valores de evapotranspiración total para el área irrigada para 12 feb 2004

\begin{tabular}{|c|c|c|c|c|c|c|}
\hline Superficie & $\begin{array}{c}\text { ET min } \\
(\mathrm{mm})\end{array}$ & $\begin{array}{c}\text { ET max } \\
(\mathrm{mm})\end{array}$ & $\begin{array}{c}\text { ET prom } \\
(\mathrm{mm})\end{array}$ & $\begin{array}{c}\text { ET Desv } \\
(\mathrm{mm})\end{array}$ & Area ha & $\begin{array}{c}\text { ET total } \\
\left(10^{3} \mathrm{~m}^{3}\right)\end{array}$ \\
\hline Mar + ríos & 4,196 & 4,2432 & 4,22 & 0,6 & 2878,99 & 121,482 \\
\hline Cultivos & 0,6 & 4,16 & 1,6 & 2,32 & 26809,51 & 428,952 \\
\hline Total & & & & & & 550,434 \\
\hline
\end{tabular}

Los valores máximos de ET son controlados por la ET asignada para el píxel frío. Sin embargo nótese que algunos píxeles tomaron valores de ET mayores, porque $\mathrm{ET}_{\text {frio }}$ representa un valor promedio de ET para toda la zona con cobertura vegetal y en buenas condiciones de riego; por lo tanto se espera que algunos campos pueden tener más ET que el píxel frío, sobre todo en áreas donde la irrigación se realizó durante el paso del satélite.

La hipótesis de píxel frío para la calibración de la imagen entera es crucial porque la selección del píxel frío "controla" la tendencia de los máximos valores de ET. Por eso, la correcta selección del píxel frío, así como el correcto cálculo de $\mathrm{ET}_{\text {frio }}$ son críticos para obtener los valores estimados de ET lo más reales posibles. La selección del píxel caliente es además importante porque esto controla y define el límite menor de los valores de ET en el área. Se observa una mayor variabilidad de los valores de ET en la zona de cultivos, debido fundamentalmente a que la tasa de ET será en función del estado hídrico y desarrollo del cultivo, así, en cultivos de regadío, existirá mayor variabilidad en función de la fecha y la cantidad de agua de riego suministrada. Esto no se espera en cultivos de secano, en los cuales se prevé una mayor concentración de valores de ET en torno a la media.

\section{Conclusiones}

La metodología permite la estimación de la evapotranspiración en áreas grandes y con su correspondiente variación espacial. La evaporación en cuerpos de agua de la imagen fue estimada como $121,482 \times 10^{3} \mathrm{~m}^{3}$.

El volumen de agua de uso consuntivo en áreas irrigadas fue estimado en $428,952 \times 10^{3} \mathrm{~m}^{3}$ por integración espacial de los valores de ET de toda el área, donde se contabilizó $26.809,51$ ha de tierras cultivadas.

Se han encontrado diferencias evidentes de albedo, temperatura y evapotranspiración según el tipo de cultivo y su estado hídrico. En concreto, los resultados muestran posibilidades de discriminar los cultivos y los estados de riego, tomando como base fundamentalmente la banda térmica de la imagen.

El uso del modelo SEBAL se ve prometedor por la ventaja de requerir pocos parámetros experimentales y su aplicación en el Perú podría ser relevante en la programación de riegos. En estudios posteriores es conveniente incluir la variable orientación del terreno en un análisis riguroso del balance de energía en zonas de laderas o montañosas. Si el análisis se realiza en zonas planas podría omitirse.

Los encargados de la gestión de los sistemas hidráulicos deberían construir un modelo operacional de sensor remoto para el cálculo de ETP en áreas grandes, para contribuir a una mejor gestión del recurso hídrico en el país.

\section{Literatura citada}

Allen, R.G.; Morse, A.; Tasumi, M.; Trezza, R.; Bastiaanssen, W.; Wright, J.L. y Kramber, W. 2002. Evapotranspiration from a satellite-based surface energy balance for Snake Plain aquifer in Idaho. Presented at the meeting of Unite States Committee on Irrigation, Drainage, and Food Control at San Luis Obispo, CA.

Allen, R.G.; Pereira, L.S.; Raes, D. y Smith, M. 1998. Crop evapotranspiration-Guidelines for computing crop water requirements. FAO, Irrigation and drainage, Paper 56. Rome, Italy: FAO.

Bastiaanssen, W.G.M. 1995. Regionalization of Surface Flux Densities and Moisture Indicators in Composite Terrain: A Remote Sensing Approach under Clear Skies in Mediterranean Climates. Ph. D. Dissertation. Wageningen: Wageningen Agricultural University.

Bastiaanssen, W.G.M. y Menenti, M. 1989. Surface Reflectance and Surface Temperature in relation with soil type and regional energy fluxes. In: A.F.

Bastiaanssen, W.G.M. (1982). Regionalization of surface flux densities and moisture indicators in composite terrain. A remote sensing approach under clear skies in Mediterranean climates. (Doctoral thesis). Wageningen Agricultural University, Wageningen, Holanda.

Brutsaert, W. Evaporation into the Atmosphere. D. Reidel.

Nelson; Allen, Rg.; Tasumi, M.; Trezza, R. y Bastiaanssen, WGM. 2002. Surface energy balance algorithms for land, Idaho implementation. Formación avanzada y manual de usuario. USA: NASA EOSDIS/ Synergy grant from the Raytheon Company through The Idaho Department of Water Resources. 